\title{
On effect of precession-induced flows in the liquid core for early Earth's history
}

\author{
S. L. Shalimov ${ }^{1,2}$ \\ ${ }^{1}$ Institute of Physics of the Earth, Moscow, Russia \\ ${ }^{2}$ Space Research Institute, Moscow, Russia
}

Received: 13 March 2006 - Revised: 12 June 2006 - Accepted: 18 September 2006 - Published: 4 October 2006

\begin{abstract}
Secondary and tertiary flow patterns seen in experiments simulating flow in the Earth's liquid core induced by luni-solar precession of the solid mantle (Vanyo et al., 1995) hint at the development of non-axisymmetric columnar periodic structures. A simple interpretation of the structure formation is presented in a hydrodynamic approach. It is suggested that if similar flow patterns can occur in the Earth's liquid core enclosed into precessing and rotating mantle then kinematic of the flows may be regarded as a possible geodynamo mechanism for early Earth's history (before the solid core formation).
\end{abstract}

\section{Introduction}

The Earth precesses with a period of about 26000 years, due to the gravity fields of the Moon and the Sun acting on the Earth's equatorial bulge of the mantle. It is a commonly accepted hypothesis that buoyancy powers stellar and planetary (for example, the Earth) dynamos while precession has a weak influence on the Earth's outer core flows and geodynamo. From recent experiments (Vanyo et al., 1995; Vanyo and Dunn, 2000) we know, however, that flows in precessing containers (liquid-filled spheroids with the Earth's like ellipticity) can become unstable and even turbulent. The flow data illustrate the real consequences of precession and ellipticity, which together with hypothetical thermal and compositional convection may give insight to direct knowledge of the Earth's deep interior and analytical solution of the geodynamo problem.

Renewing interest to the problem of precession-driven geodynamo is stimulated by the attempts to reconcile the energy requirement of the convective dynamo that puts con-

Correspondence to: S. L. Shalimov

(shalimov@ifz.ru) straints on the thermal budget and evolution of the core through Earth's history.

The situation under discussion is the following. The limited thermodynamic efficiency of thermal convection needs in addition a compositional convection (driven by rejection of light alloying elements from the growing solid inner core) to produce the observed geomagnetic field. Rapid core cooling favours the operation of a geodynamo but creates an inner core size that is too large in comparison with the present-day radius. Slower cooling reduces the inner core size but makes a geodynamo less likely to operate. Introducing radioactive potassium into the core retards inner core growth and provide an additional source of entropy. But even the last model (keeping the correct present-day inner core radius) has an inner-core age of $<1.5 \mathrm{Gyr}$ (Nimmo et al. 2004). It was supposed that prior to that time the geodynamo was probably sustained by a mixture of radioactive decay and cooling in an entirely liquid core. However the existence of a magnetic field of roughly present-day strength over the past $3.5 \mathrm{Gyr}$ (McElhinny and Senanayake, 1980) means that thermal convection at work with a very high cooling rate which implies very high temperature and a partially molten lower mantle in the distant past (Gubbins et al., 2003). Such a boundary condition in the lower mantle could result in radical effects for tectonic history of the Earth. Thus, there is a need for another energy source other than merely convection in order to generate geomagnetic field during $2 \mathrm{Gyr}$ before the inner core formation.

Traditionally precession is considered as an alternative of convection (Malkus, 1968; Dolginov, 1995). The solution of the equation of motion for inviscid fluid in a precessing ellipsoidal container consists of elliptical streamlines confined to a plane (Sloudsky, 1895; Poincare, 1910) and presents a kind of solid body rotation. The solution of the inviscid problem is not unique, and the laminar precession driven flow of viscous fluid has been analytically constructed (Stewartson and Roberts, 1963; Busse, 1968).

Published by Copernicus GmbH on behalf of the European Geosciences Union and the American Geophysical Union. 
Malkus (1968) was the first who advanced detailed experimental and theoretical arguments to support a geodynamo driven by luni-solar precession. He used an oblate rotating and precessing spheroid and based his analytical consideration (the power estimate of precession-driven geodynamo that turns out to be $2 \times 10^{10} \mathrm{~W}$ and is about two order of magnitude less than present-day estimations) on the observed hydromagnetic turbulence in the liquid of the spheroid. Critiques of this idea (Rochester et al., 1975; Loper, 1975) focused upon establishing the energetic irrelevance of the laminar geodynamo response to the precession rather than considering the fully turbulent case. Meanwhile Kerswell (1993) derived criteria for the onset of turbulence in precession and then calculated (Kerswell, 1996) upper bound on the energy dissipation in turbulent precession. He advocated that a dissipation of $10^{21} \mathrm{~W}$ may potentially be realized in a fully turbulent outer core, which is about 14 orders of magnitude more than well-known laminar value of $10^{7} \mathrm{~W}$ (Loper, 1975) conventionally assumed.

Thus, the statement about energetic irrelevance of geodynamo response to the precession which dominated during 30 years should be transformed into a question: whether precession can drive turbulence and/or flows that has a structure suitable for magnetic field generation? The aim of the paper is a clarification of a positive answer on the second part of the question using analytical approach. As a consequence of this consideration we suggest the leading role of precessiondriven geodynamo in early Earth's history.

\section{Structure of flows in laboratory and interpretation}

The Earth is approximately an oblate spheroid with symmetry axis and spin axis nearly aligned with the north-south direction. The surface ellipticity is about $1 / 298$, and the ellipticity at the boundary of the solid mantle and liquid core is about $1 / 400$. The Earth equatorial bulge of the mantle is inclined by $23.5^{\circ}$ to the orbital plane. As a result the solid mantle is subject to gravitational torques from the sun and moon so that the complete precession takes approximately 26000 years. It is supposed that the liquid core does not respond directly to the luni-solar torques, but rather can interact with the spinning and precessing mantle. The Earth ratio of spin period ( 1 day) to precession period $(26000 \mathrm{yr})$ - the so-called Poincare number - is $10^{-7}$. The ratio of viscous to Coriolis force - the so-called Ekman number which to some extent is the characteristic of coupling between the liquid core and the mantle at the core-mantle boundary - is highly uncertain for the Earth's liquid core (due to uncertainty of the viscosity) so usually the Ekman number takes the range $10^{-15} \div 10^{-6}$.

Specifically, in the first investigations of precessioninduced flow in oblate spheroid by Malkus (1968) the ellipticity of containers took values $1 / 10$ and $1 / 25$. The last experiments by Vanyo et al. (1995) and Vanyo and Dunn (2000) have included spheroids with ellipticities 1/100 and
1/400. Minimal Poincare number achieved in experiments by Malkus and Vanyo et al. was $1 / 80$ and $10^{-6}$, and Ekman number was $3.6 \times 10^{-6}$ and $2 \times 10^{-7}$, respectively. In a theoretical analyses below we only require for Ekman number that $E k \ll 1$.

Precessional flows turned out to be extremely sensitive to precession rate and ellipticity. That is why experimental results obtained by Vanyo et al. (1995) and Vanyo and Dunn (2000) differ significantly from that of Malkus (1968). Specifically, Vanyo et al. have been able to match the Earth's parameters more closely than in previous experiments. The extension of the experiments to smaller Poincare numbers in particular has made it possible to characterize a laminar regime that corresponds in principle to that of the Earth and to identify the various basic constituent flows in this regime. The results reported by Vanyo's group raise some doubt about Malkus's conclusions based on turbulent state of the core.

The following flow features have been identified in Vanyo et al. (1995) and Vanyo and Dunn (2000) experiments: 1) A uniform rotation component with an angular rotation vector deviating (at a very small angle) from the container's rotation axis. The fluid spin axis precesses at the same rate as the container spin axis, but with a lag. 2) A secondary flow consisting of nested cylindrical shear layers with axes roughly parallel to the fluid spin axis. These cylindrical layers rotate alternately faster and slower than the fluid as a whole. 3) Finally, the possibility of axially parallel columns with alternating vorticity similarly to the buoyancy convection patterns depicted by Busse (1970) has been recognized as a tertiary flows. It should be noted that patterns starting as a surface flows after some time progressed axially into the interior. These slowly evolving waveforms represent nonaxisymmetric motion of the fluid, the type of flows believed to be critical for maintenance of the geodynamo.

The phenomena mentioned above are shown schematically in Fig. 1 (adapted from Vanyo et al., 1995).

To interpret the appearance of the tertiary flows in the experiment the incompressible fluid of density $\rho$ and kinematic viscosity $v$, in a spherical shell of radius $R$ rotating with angular frequency $\omega_{d}$ about symmetry axis is considered. Spin axis deviates at an angle $\alpha$ from the vertical axis. The shell executes precessional motion characterized by the precessional vector $\omega_{p}$. The equation of motion reads in the frame of reference attached to the shell (mantle) (Pedlosky, 1982)

$$
\begin{aligned}
& \frac{\partial \boldsymbol{v}}{\partial t}+(\boldsymbol{v} \nabla) \boldsymbol{v}+2\left(\omega_{d}+\omega_{p}\right) \times \boldsymbol{v}=-\frac{1}{\rho} \nabla p+v \Delta \boldsymbol{v}-\left(\omega_{p} \times \omega_{d}\right) \times \boldsymbol{r}, \\
& \nabla \cdot \boldsymbol{v}=0 .
\end{aligned}
$$

Note that a reduced version of pressure $p$ is not written here, and it will not appear in the following.

Using evidence from experiments (Vanyo et al., 1995; Vanyo and Dunn, 2000) we consider the background flow structure with differentially rotating cylinders (shear 


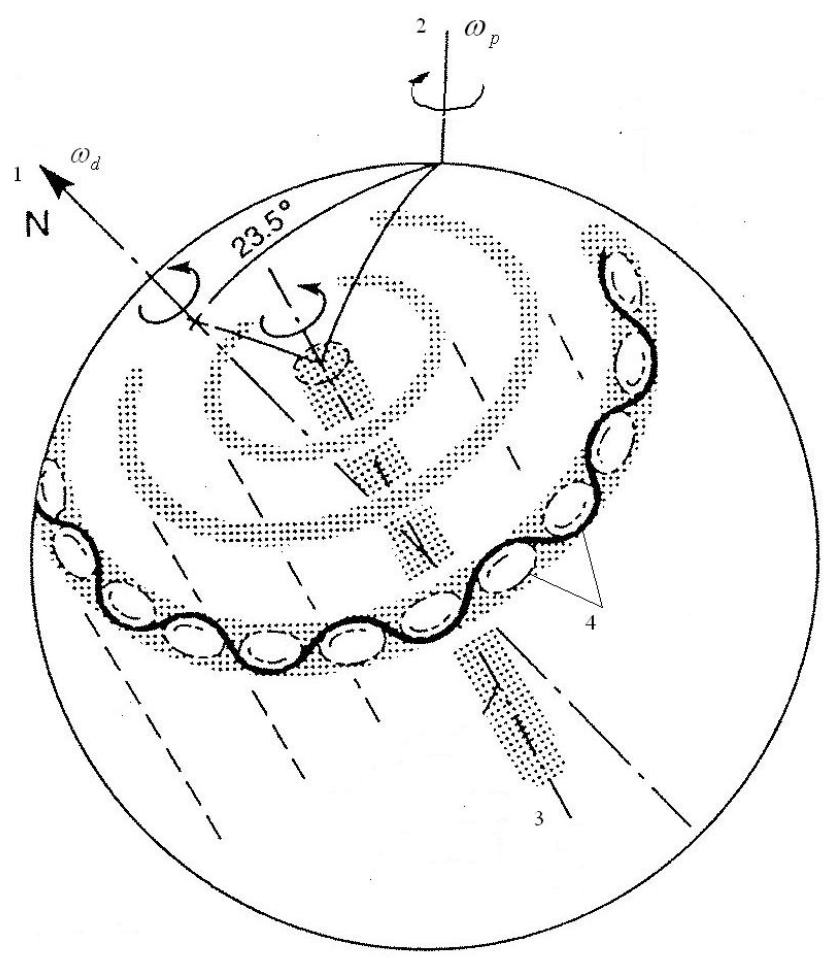

Fig. 1. Schematic view of flows (not to scale) observed in experiments with rotating and precessing container (adapted from Vanyo et al., 1995): 1 - container spin axis, 2 - precession axis, 3 - fluid core spin axis, 4 - typical flows.

structure), and ignore for simplicity a deviation of fluid angular rotation from the container's rotation axis.

Taking the curl of Eq. (1) and making then a projection on rotation $z$ axis in $\beta$-plane approximation one obtains

$\frac{\partial \omega}{\partial t}+[\psi, \omega]+\beta \frac{\partial \psi}{\partial x}=v \Delta \omega$

where $\omega=\Delta \psi$ is the fluid vorticity, $\psi$ is the twodimension stream function, $\mathrm{v}_{x}=-\partial \psi / \partial y, \quad \mathrm{v}_{y}=\partial \psi / \partial x$, $[a, b]=(\partial a / \partial x)(\partial b / \partial y)-(\partial a / \partial y)(\partial b / \partial x)$ is the Jacobian of $a$ and $b$, parameter $\beta=2 \partial\left(\omega_{d}+\omega_{p}\right)_{z} / \partial y$ takes into account differential rotation, $x, y$ are the eastward and northward axes, respectively.

Existence of Ekman layer at fluid-shell boundary is similar to a weak stratification in shear flow of incompressible fluid (Howard, 1963). If characteristic scales of vortex structures are larger than Ekman layer thickness, the flows can be considered as two-dimensional. Neglecting the viscous effect let us consider solution to Eq. (2) in the form $\psi=\psi(\xi, y), \quad \xi=x-u t$, where $u$ is the velocity along the parallel ( $x$-axis). Then Eq. (2) becomes

$\left[\omega+2\left(\omega_{d}+\omega_{p}\right)_{z}, \psi+u y\right]=0$,

and one finds

$\Delta \psi=-2\left(\omega_{d}+\omega_{p}\right)+f(\psi+u y)$.

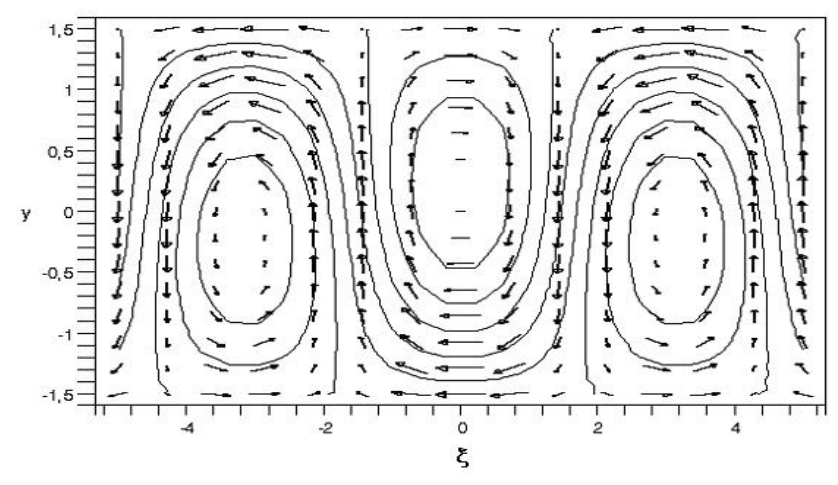

Fig. 2. Streamline pattern for counter-rotating row of vortices in the reference frame traveling with velocity $u$.

Here $f=f(\eta)$ is an arbitrary function, which we take in the form (Hasegawa, 1985)

$f(\eta)=-k^{2}(\eta)$.

So we may rewrite Eq. (3) as

$\Delta(\psi+u y)+2\left(\omega_{d}+\omega_{p}\right)=-k^{2}(\psi+u y)$.

Well-known solution to this equation is

$\psi+u y=\frac{2\left(\omega_{d}+\omega_{p}\right)}{k^{2}}+\psi_{1}, \quad \Delta \psi_{1}=-k^{2} \psi_{1}$

which means vortices with a scale of $\lambda=2 \pi / k$ imposed on a zonal flow. The simplest solution of equation for $\psi_{1}$ can be represented as $\psi_{1}=C \cos k_{x} \xi \cos k_{y} y$, where $C$ is the amplitude and $k_{x}^{2}+k_{y}^{2}=k^{2}$. Corresponding flow structure with large amplitude and with a wavelength of the order of meridional scale is shown in Fig. 2.

\section{Discussion and conclusions}

According to experiment (Vanyo et al., 1995) the meridional scale of a zonal flow developed in precessing and rotating container corresponds to a few hundreds km of the Earth's liquid core. It is seen from Fig. 2 that in the coordinate system traveling with velocity $u$ the solution is a row of vortices of alternating sign - cyclonic and anticyclonic. Mechanism of Ekman pumping acting throughout the boundary layer (fluid-shell boundary) forces liquid to move in opposite directions inside cyclonic and anticyclonic regions. This results in fluid circulation indicated in Fig. 3a when helical flows are organized into columns that extend across the container in the direction of the rotation axis. This reminds columnar eddies observed in buoyancy-driven convection in rotating spheres (Busse, 1970), and is also in agreement with precession-driven patterns observed by Vanyo et al. (1995) and Vanyo and Dunn (2000). It should be stressed here that while a vortex chain formation can be explained by taking $E k=0$, both the formation of zonal flows and columnar 


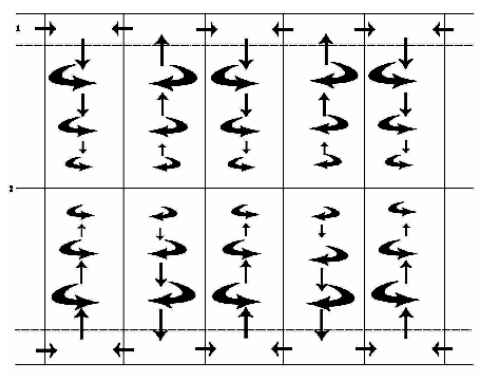

(a)

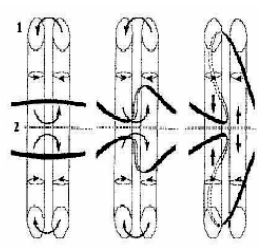

(b)
Fig. 3. (a) columnar eddies development from a row of counterrotating vortices (1 - Ekman layer, 2 - equator); (b) poloidal magnetic field component generated from toroidal component with flows shown in panel (a).

patterns progressed into the interior of fluid (due to Ekman pumping mechanism) need $E k \neq 0$.

Such a flow structure, shown in Fig. 3a, can operate as a kinematic dynamo. In Fig. 3b, adapted from (Kageyama and Sato, 1997), arrows indicate the direction of flow. An initially zonal (toroidal) magnetic field (left) is represented by two thick lines on either side of the equator (dashed line). The helical flow distorts the initial field configuration to produce loops of poloidal field that are perpendicular to the initial field (right). Just the same mechanism of $\alpha^{2}$-dynamo has been used to interpret dipole magnetic field generation in numerical calculations (Kageyama and Sato, 1997).

If precession plays a significant role in the generation of the magnetic field, paleomagnetic measurements must contain information about the influence of precession on the geomagnetic field. For example, Channell et al. (1998) presented evidence for a strong correlation between magnetic paleointensity records and variations of orbital obliquity (the angle $\alpha$ varying from $22^{\circ}$ to $24.5^{\circ}$ ) which affect precesssional forces in the core. Indeed, the energy dissipation at the sphere-cavity interface (core-mantle boundary in case of the Earth) in the frame of rigid-body model with viscous coupling (see, for example, Vanyo and Dunn, 2000) is proportional to $\sin ^{2} \alpha$. On the other hand, this dependence should affect the geomagnetic field stability. For example, it is known from the convective geodynamo theory (Jones, 2000; Reshetnyak, 2005) that a weakening of the geomagnetic field reduces the flow scale in the Earth's liquid core (in the plane perpendicular to the rotation axis). This leads to a more rapid rate of their ohmic dissipation, and the further generation of the field stops. The recovery of the previous field intensity is apparently due to nonlinear effects (see, for example, Shalimov, 2004). With the combined action of the convective and precession driven geodynamo, this scenario of the magnetic field stability is quite possible. In other words, excursions and reversals of the geomagnetic field must correlate with low precession amplitude (low $\alpha$ ).
Finally, the two parts of the flows observed with precessing and rotating containers by Vanyo et al. (1995) and Vanyo and Dunn (2000) - large-scale axisymmetric zonal shears and smaller-scale columnar patterns - are the same basic elements observed in buoyancy-driven convection in rotating spheres. It has been demonstrated that the columnar patterns can somehow be related with zonal shears. Accordingly, such precessional flows acting in the Earth's liquid core can, in principle, produce a kinematic dynamo similar to a convection driven dynamo. (We note the recent numerical results in this respect - Tilgner, 2005). This precession-driven geodynamo mechanism possibly operated through the early Earth's history (before the solid core formation).

Acknowledgements. Support from RFBR (grant no. 04-05-64862) is gratefully acknowledged.

Edited by: N. S. Erokhin

Reviewed by: three referees

\section{References}

Busse, F. H.: Steady fluid flow in a precessing spheroidal shell, J. Fluid. Mech., 33, 739-751, 1968.

Busse, F. H.: Thermal instabilities in rapidly rotating systems, J. Fluid. Mech., 44, 441-460, 1970.

Channell, J. E. T., Hodel, D. A., McManus, J., and Lehman, B.: Orbital modulation of the Earth's magnetic field intensity, Nature, 394, 464-468, 1998.

Dolginov, Sh. Sh.: Precession of planets and generation of their magnetic fields, Geomagnetism and Aeronomy (in Russian), 35, 1-29, 1995.

Gubbins, D., Alfe, D., Masters, G., et al.: Can the Earth's dynamo run on heat alone?, Geophys. J. Int., 155, 609-622, 2003.

Hasegawa, A.: Self organization processes in continuous media, Adv. Phys., 34, 1-42, 1985.

Howard, L. W.: Neutral curves and stability boundaries in stratified flows, J. Fluid. Mech., 16, 333-342, 1963.

Jones, C. A.: Convection-driven geodynamo models, Phil. Trans. R. Soc. London, A358, 873-884, 2000.

Kageyama, A. and Sato, T.: Generation mechanism of a dipole field by a magnetohydrodynamic dynamo, Phys. Rev. E., 55, 46174626, 1997.

Kerswell, R. R.: The instability of precessing flow, Geophys. Astrophys. Fluid Dyn., 72, 107-144, 1993.

Kerswell, R. R.: Upper bounds on the energy dissipation in turbulent precession, J. Fluid. Mech., 321, 335-370, 1996.

Labrosse, S.: Thermal and magnetic evolution of the Earth's core, PEPI, 140, 127-143, 2003.

Loper, D.: Torque balance and energy budget for the precessionally driven dynamo, PEPI, 11, 43-60, 1975.

Malkus, V. W. R.: Precession of the Earth as a cause of geomagnetism, Science, 160, 259-264, 1968.

McElhinny, M. W. and Senanayake, W. E.: Paleomagnetic evidence for the existence of the geomagnetic field $3.5 \mathrm{Ga}$ ago, J. Geophys. Res., 85, 3523-3528, 1980. 
Nimmo, F., Price, G. D., Brodholt J., and Gubbins, D.: The influence of potassium on core and geodynamo evolution, Geophys. J. Int., 156, 363-376, 2004.

Pedlosky, J.: Geophysical Fluid Dynamics, Springer-Verlag, New York, 1982.

Poincare, H.: Sur la precession des corps deformables, Bull. Astron., 27, 321-356, 1910.

Reshetnyak, M. Yu.: Dynamo catastrophe, or why geomagnetic field exists so long?, Geomag. Aeronom. (in Russian), 45, 571575, 2005.

Rochester, M. G., Jacobs, J. A., Smylie, D. E., and Chong, K. F.: Can precession power the geomagnetic dynamo?, Geophys. J. R. Astr. Soc., 43, 661-678, 1975.

Shalimov, S. L.: On instability of geomagnetic field, Dokl. Akad. Nauk (in Russian), 395, 258-260, 2004.
Sloudsky, Th.: De la rotation de la terre suppose fluide a son interieur, Bull. De la Soc. Impeiale des Naturalistes., 9, 285-318, 1985.

Stewartson, K. and Roberts, P. H.: On the motion of a liquid in a spheroidal cavity of a precessing rigid body, J. Fluid Mech., 17, 1-20, 1963.

Tilgner, A.: Precession driven dynamos, Phys. Fluids, 17, 0341041-034104-6, 2005.

Vanyo, J., Wilde, P., Cardin, P., and Olson, P.: Experiments on precessing flows in the Earth's liquid core, Geophys. J. Int., 121, 136-142, 1995.

Vanyo, J. and Dunn, J. R.: Core precession: flow structures and energy, Geophys. J. Int., 142, 409-425, 2000. 